\title{
MicroRNA-499 rs3746444 A/G polymorphism functions as a biomarker to predict recurrence following endoscopic submucosal dissection in primary early gastric cancer
}

\author{
HUIYONG SHI, XIANGSHAN YANG, YANAN ZHEN, SHOUJUN HUO, RUIXUE XIAO and ZHONGFA XU \\ Department of General Surgery, Affiliated Hospital of Shandong Academy of Medical Sciences, \\ Jinan, Shandong 250031, P.R. China
}

Received September 23, 2015; Accepted October 12, 2016

DOI: $10.3892 / \mathrm{mmr} .2017 .6369$

\begin{abstract}
The aim of the present study was to investigate the molecular mechanism, including the potential regulatory and signaling pathways, of platelet-derived growth factor receptor $\beta$ (PDGFRB), which underlies the recurrence of early gastric cancer (EGC) following endoscopic submucosal dissection (ESD). Online microRNA (miRNA) target prediction tools were used, which identified PDGFRB as the candidate target gene of miR-499a in gastric cancer cells, and PFGRBR was then confirmed as the direct gene using a luciferase reporter assay system. The Kaplan-Meier method was used to plot recurrence-free curves, which were compared between genotype groups. A negative regulatory association between miR-499a and PDGFRB was established by investigating the relative luciferase activity at different concentrations of miR-499a mimics. Furthermore, as the rs 3746444 polymorphism has been previously reported to interfere with the expression of miR-499a, the present study investigated the expression levels of different genotypes, including TT $(n=20)$, TC $(n=9)$ and CC $(n=3)$, the results of which supported the hypothesis that the presence of the minor allele (C) of the rs3746444 polymorphism compromised the expression of miR-499a. The present study also performed polymerase chain reaction and western blot analyses to examine the mRNA and protein expression levels of PFGRBR among different genotypes or cells treated with different concentrations of miR-499a mimics/inhibitors, which indicated the negative regulatory association between miR-499a and PDGFRB. The present study also investigated the relative viabilities of EGC cells transfected with miR-499a mimics (50 and $100 \mathrm{nM}$ ) and miR-499a inhibitors (100 nM), and confirmed that miR-499a negatively interfered with the
\end{abstract}

Correspondence to: Dr Zhongfa Xu, Department of General Surgery, Affiliated Hospital of Shandong Academy of Medical Sciences, 38 Wuyingshan Road, Jinan, Shandong 250031, P.R. China E-mail: esdrecurrence@163.com

Key words: microRNA-499a, rs3746444, biomarker, recurrence, endoscopic submucosal dissection, early gastric cancer viability of the EGC cells. The miR-499a rs3746444 polymorphism was also recognized as a biomarker to predict recurrence following ESD in patients with EGC via analyzing the recurrence-free rates among patients with EGC with different genotypes. The results showed that PDGFRB was validated as a target of miR-499a, and rs3746444 was identified as a potential biomarker to predict the recurrence of EGC following ESD.

\section{Introduction}

Gastric cancer is the second most common malignancy in the world; however, the outcome of early gastric cancer (EGC) has markedly improved (1). An increasing number of patients with EGC receive endoscopic treatment and benefit from an improvement in their prognosis; consequently, laparotomy is avoided and an improved quality of life is maintained in several patients suffering from EGC (2). Endoscopic resection as the standard therapy for EGC is widely accepted and regularly used worldwide.

Previously, Ono et al (3) developed an innovational technique, endoscopic submucosal dissection (ESD), which has become a well-accepted technique for the treatment of EGC, and pathological examination provided by the ESD method enables accurate diagnosis (4). ESD is associated with a good prognosis of EGC $(5,6)$, however, multiple EGCs may occur following curative resection, with reports demonstrating that 6-14\% of patients with EGC deteriorate (7-9). Consequently, it is difficult to determine the optimal follow-up strategy, which aims at identifying recurrences of EGC following ESD, as there is no suitable biomarker to predict recurrence.

MicroRNAs (miRNAs) are a group of hairpin-derived RNAs consisting of 20-24 nucleotides, which bind to the 3' untranslated region (3'UTR) of messenger RNA (mRNA), resulting in post-transcriptional repression of the expression of target genes in disease and normal physiological contexts in a broad range of organisms $(10,11)$. Typically, nuclear RNase III (Drosha) cleaves the long primary precursors (pri-miRNAs) into pre-miRNAs of $\sim 70 \mathrm{nt}$ in length following transcription mediated by RNA polymerase II. A stem-loop structure is present in the resulting transcripts, which are then exported to the cytoplasm, followed by processing by 
RNase III (Dicer), generating mature double-stranded $~ 22 \mathrm{nt}$ miRNAs (12). The miRNA-target interaction may be altered or the maturation process of miRNA may be affected when genetic variation occurs in the pre-miRNA sequence. Several studies have indicated that the risk of cancer or pre-cancer may be increased by the presence of miRNA polymorphisms (13); however, few have examined the association between cancer susceptibility and miRNA polymorphisms. The arm selection of miR-499a or the target selection of miRNA may be altered directly by the rs 3746444 in the seed region of the miR-499a (14).

The present study aimed to investigate the molecular mechanism, including the potential regulatory and signaling pathways, of platelet-derived growth factor receptor $\beta$ (PDGFRB), which underlies the recurrence of EGC. PDGFRB was identified as a target of miR-499a. As the expression of PDGFRB has been reported to be associated with EGC recurrence following ESD (15), and the rs3746444 polymorphism may alter the expression of miR-499a, it was hypothesized that the presence of rs3746444 may be associated with the recurrence of EGC following ESD via targeting PDGFRB.

\section{Materials and methods}

Patients and specimens. A total of 128 patients (84 males and 44 females; mean age, 57 years; range, 26-80 years), who received ESD for EGC in the Affiliated Hospital of Shandong Academy of Medical Sciences (Jinan, China) between September 2013 and September 2014, were included in the present study. The EGC was defined as adenocarcinoma confined to the submucosa or mucosa of the stomach. Patients who had received ESD or multiple ESDs previously were excluded from the investigation. Peripheral blood was collected from all 128 patients, tissue samples $(\sim 0.5 \mathrm{~cm})$ were available from 32 patients for RNA extraction and western blot analysis. The duration between the first dose of ESD and the final dose was defined as the follow-up period. Following the first dose of ESD, patients received follow-up ESDs up to 42 months. Patient clinical information, including age, gender, smoking history, body mass index, tumor location, grade of gastric atrophy, tumor differentiation and Helicobacter pylori infection status at the time of first dose of ESD, were obtained based on medical records. All subjects signed written informed consent prior to enrollment, and the study protocol was approved by the Ethics Committee of Shandong Academy of Medical Sciences. The principles of the declaration of Helsinki were complied with in the present study.

Polymorphism genotyping. Genomic DNA was extracted and purified from the whole-blood samples with a QIAamp DNA midi kit (Qiagen Sciences, Inc., Germantown, MD, USA). The TaqMan real-time polymerase chain reaction (PCR) method and the ABI PRISM 7500 sequence detection system (Applied Biosystems; Thermo Fisher Scientific, Inc., Waltham, MA, USA) were used to analyze the genotypes of miR-499a T $>C$ (rs3746444) in a 96-well format primer used was 5'-AAACAU CACUGCAAGUCUUAA-3'. The PCR analysis was performed using $10 \mu \mathrm{l}$ reaction mixture of $\mathrm{ddH}_{2} \mathrm{O}, 0.25 \mu \mathrm{l}$ primer/probe mixture, $5 \mu \mathrm{l}$ TaqMan Universal PCR Master mix (Applied
Biosystems; Thermo Fisher Scientific, Inc.) and $20 \mathrm{ng}$ DNA using the following thermocycling conditions: $94^{\circ} \mathrm{C}$ or $10 \mathrm{~min}$, followed by 40 cycles at $94^{\circ} \mathrm{C}$ for $20 \mathrm{sec}$ and $60^{\circ} \mathrm{C}$ for $45 \mathrm{sec}$. In order to determine the genotype of samples, SDS v1.3.1 software (Applied Biosystems; Thermo Fisher Scientific, Inc.) was used to analyze the allele-specific fluorescence data in each plate.

$R N A$ extraction and stem-loop reverse transcription-PCR (RT-PCR) analysis. TRIzol reagent (Invitrogen; Thermo Fisher Scientific, Inc.) was used to extract total RNA from the 32 tissue samples. In brief, the tissue samples were homogenized with $1 \mathrm{ml}$ TRIzol reagent, and $0.2 \mathrm{ml}$ chloroform was added to extract proteins. Isopropanol $(0.5 \mathrm{ml})$ was added to precipitate the RNA. A Nanodrop 1000 spectrophotometer (Nanodrop Technologies, Inc.; Thermo Fisher Scientific, Inc., Wilmington, DE, USA) was used to determine the quantity, purity and concentration of total RNA. A reaction mixture comprising a stem-loop RT, SuperScript III Reverse Transcriptase (Invitrogen; Thermo Fisher Scientific, Inc.), and RT primers for miR-499a and PDGFRB mRNA were used for reverse transcription of the total RNA. The following primers were used: $\beta$-actin forward, 5'-GTCATTCCAAATATGAGA TGCGT-3' and reverse, 5'-GCATTACATAATTTACACGAA AGCA-3', RNU6 forward, 5'-CTCGCTTCGGCAGCACA-3' and reverse, 5'-AACGCTTCACGAATTTGCGT-3'. The reaction conditions were as follows: $16^{\circ} \mathrm{C}$ for $30 \mathrm{~min}$, followed by $30 \mathrm{sec}$ at $20^{\circ} \mathrm{C}, 30 \mathrm{sec}$ at $42^{\circ} \mathrm{C}, 1 \mathrm{sec}$ at $50^{\circ} \mathrm{C}$ for 50 cycles. Finally, the reaction mixture was incubated at $85^{\circ} \mathrm{C}$ for $5 \mathrm{~min}$ to inactivate the enzyme. A SYBR Green I assay (Applied Biosystems; Thermo Fisher Scientific, Inc.) using the following thermocycling conditions: $95^{\circ} \mathrm{C}$ for $30 \mathrm{sec}, 95^{\circ} \mathrm{C}$ at $3 \mathrm{sec}$ for 40 cycles, $60^{\circ} \mathrm{C}$ for $15 \mathrm{sec}$ to detect gene expression. The mRNA expression level of PDGFRB and the expression level of miR-499a were normalized to that of U6 (2 $\left.2^{-\Delta \Delta \mathrm{Cq}}\right)(16)$.

Cell culture and oligonucleotide transfection. MKN-45 cells were obtained from American Type Culture Collection (Manassas, VA, USA). The cells were cultured in RPMI-1640 supplemented with streptomycin and penicillin, and FBS (Gibco; Thermo Fisher Scientific, Inc.). The miR-499a mimics and inhibitors were synthesized by Sangon Biotech Co., Ltd. (Shanghai, China), and Lipofectamine 2000 (Invitrogen; Thermo Fisher Scientific, Inc.) was used to perform the transfection, cells were seeded at density of $8 \times 10^{6}$ cells/well at room temperature for 10-15 min. The cells were collected for the proliferation assay $24 \mathrm{~h}$ following transfection, and at $48 \mathrm{~h}$ post-transfection, the cells were harvested for the analyses of protein, RNA and cell viability. MiRanda (www.microrna.org) and TargetScan (www.targetscan.org) were used in the present study.

Luciferase reporter assay. The 3'UTR luciferase assay was performed using a Psicheck ${ }^{\mathrm{TM}}$-2 Dual-Luciferase miRNA target expression vector (Promega Corporation, Madison, WI, USA). PCR was performed to amplify the 3'UTR sequence of the PDGFRB gene. The cDNA was then amplified by qPCR using a SYBR green qPCR kit (Takara Bio, Inc., Tokyo, Japan) in a $25 \mu 1$ with $12.5 \mu 1$ of 2 xSYBR Fast qPCR Mix (Takara Bio, Inc.), $2 \mu \mathrm{l}$ cDNA, $1 \mu \mathrm{l}$ reverse primer $(10 \mu \mathrm{m}), 1 \mu \mathrm{l}$ of 
forward primer $(10 \mu \mathrm{m})$ and $8.5 \mu \mathrm{l}$ water without nuclease. The amplification was carried out at $98^{\circ} \mathrm{C}$ for $60 \mathrm{sec}$, and followed by 30 cycles $\left(98^{\circ} \mathrm{C}\right.$ for $30 \mathrm{sec}, 58^{\circ} \mathrm{C}$ for $30 \mathrm{sec}, 72^{\circ} \mathrm{C}$ for $120 \mathrm{sec}$ ) and $72^{\circ} \mathrm{C}$ for $5 \mathrm{~min}$. The product was cloned into a Psicheck ${ }^{\mathrm{TM}}-2$ vector to generate a wild-type reporter. A Site-directed gene mutagenesis kit (Beyotime Institute of Biotechnology, Shanghai, China) was used to produce the mutant reporter, according to the manufacturer's protocol. For the luciferase assay, the Psicheck ${ }^{\mathrm{TM}}-2$ Dual-Luciferase miRNA target expression vector containing the mutant or wild-type target sequences were used to co-transfect the miR-499a-overexpressing MKN-45 cells using Lipofectamine 2000 (Invitrogen; Thermo Fisher Scientific, Inc.). At $48 \mathrm{~h}$ post-transfection, the cells were harvested and lyzed in passive lysis buffer (Promega Corporation). The firefly luciferase activity was detected in a Steady Glo Luciferase Assay system (Promega Corporation) with renilla activity as a control.

Cell viability assays. Following transfection, the cells were incubated in 96-well plates at a density of $1 \times 10^{4} \mathrm{MKN}-45$ cells per well. 3-(4,5-dimethylthiazol-2-yl)-2,5-diphenyl-2H-tetrazolium bromide was added into each well at a final concentration of $20 \mu \mathrm{l}$ of $5 \mathrm{mg} / \mathrm{ml}$ to a final volume of $250 \mu \mathrm{l}$, and the cells were then incubated at $37^{\circ} \mathrm{C}$ for $4 \mathrm{~h}$. DMSO was added to dissolve the remaining crystals following removal of the culture medium. The absorbance at $570 \mathrm{~nm}$ was measured using a microplate reader.

Immunoblot analysis. RIPA lysis buffer (Beyotime Institute of Biotechnology) was used to extract total proteins from the transfected MKN-45 cells or tissue samples according to the manufacturer's protocol. A bicinchoninic acid protein assay was used to quantify whole cell protein extract. An equivalent volume (30 $\mu \mathrm{g}$ per lane) of cell lysate was separated in a $10 \%$ SDS-PAGE gel and then transferred onto a polyvinylidene fluoride membrane. The membranes were blocked in TBST supplemented with $5 \%(\mathrm{w} / \mathrm{v})$ skimmed milk at $4^{\circ} \mathrm{C}$ for $1 \mathrm{~h}$, following which primary antibodies were incubated at $4^{\circ} \mathrm{C}$ for $12 \mathrm{~h}$ using PDGFRB (cat. no. sc-12910; 1:500; Santa Cruz Biotechnology, Inc., Dallas, Texas) and $\beta$ actin (cat. no. sc-47778; 1:1,500; Santa Cruz Biotechnology, Inc.). The enhanced chemiluminescence reagent (EMD Millipore, Billerica, USA) was used to visualize the protein bands following incubation at room temperature for $2 \mathrm{~h}$ with horseradish peroxidase-conjugated secondary antibody (cat. no. sc-516086; 1:10,000; Santa Cruz Biotechnology, Inc.). The signals on the blots were detected using an ECL kit (Amersham ECL detection system; GE Healthcare Life Sciences, Chalfont, UK), and the relative density of the target bands were analyzed using Image $\mathrm{J}$ software (National Institutes of Health, Bethesda, MD, USA).

Statistical analysis. The $\chi^{2}$-test was used to analyze the differences between discrete variables and one-way analysis of variance was used to analyze differences between continuous variables in each group. The Kaplan-Meier method was used to plot recurrence-free curves, which were compared using the log rank test. Hazard ratios were calculated using the univariate and multivariate Cox proportional hazard model. $\mathrm{P}<0.05$ was considered to indicate a statistically significant difference.

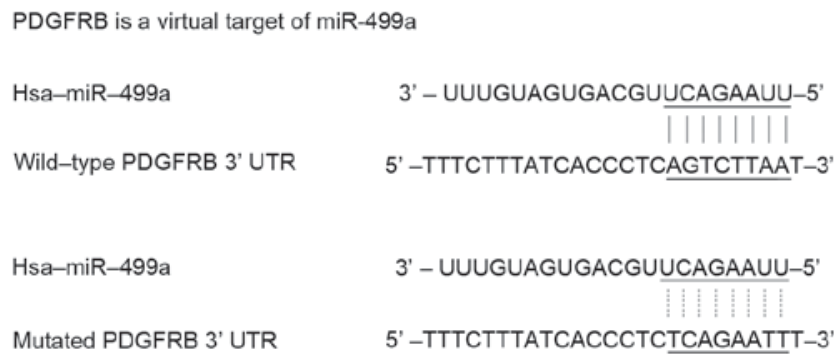

Figure 1. Identification of PDGFRB as the candidate target gene of miR-499a in gastric cancer cells with the 'seed sequence' in the 3'UTR of PDGFRB. 3'UTR 3' untranslated region; miR, microRNA; PDGFRB, platelet-derived growth factor receptor $\beta$.

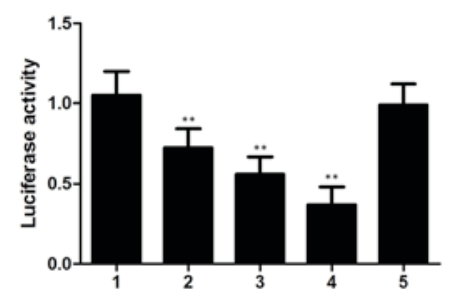

1. scramble control
2. wild-type PDGFRB $3^{\prime} U T R$
miR-499a mimic ( $25 \mathrm{nM})$
3. wild-type PDGFRB $3^{\prime} \mathrm{UTR}$
miR-499a mimic (50 nM)
4. wild-type PDGFRB $3^{\prime} \mathrm{UTR}$
miR-499a mimic (100 nM)
5. mutated PDGFRB $33^{\prime} \mathrm{UTR}$
miR-499a mimic ( $25 \mathrm{nM})$

Figure 2. Luciferase activity reporter assay verification of PDGFRB as the direct target gene of miR-499a and validation of the regulatory association between miR-449a and PDGFRB. Compared with the scramble control, the relative luciferase activity of cells transfected with wild-type PDGFRB 3'UTR constructs decreased as the concentration of miR-499a mimics increased, exhibiting a negative regulation in a step-wise manner. The cells carrying mutant PDGFRB 3'UTR constructs exhibited comparable relative luciferase activity, compared with the scramble controls, indicating PDGFRB was the direct target gene of miR-499a, with the binding site located in the mutated segment. ${ }^{* *} \mathrm{P}<0.01$ vs. control. 3'UTR 3' untranslated region; miR, microRNA; PDGFRB, platelet-derived growth factor receptor $\beta$.

Data are expressed as the mean \pm standard deviation. SPSS 18 (SPSS, Inc., Chicago, IL, USA) was used to perform all statistical analyses.

\section{Results}

PDGFRB is a target of miR-499. PDGFRB has been previously reported to be a growth factor receptor, the expression level of which affects the recurrence of EGC following ESD treatment (17). The present study aimed to investigate the molecular mechanism, including the potential regulatory and signaling pathways, of PDGFRB, which underlies the recurrence of EGC following ESD treatment. As shown in Fig. 1, online miRNA target prediction tools were used to identify the regulatory miRNA of PDGFRB, and consequently identified PDGFRB as the candidate target gene of miR-499a in gastric cancer cells with the 'seed sequence' in the 3'UTR of PDGFRB.

Furthermore, to validate the regulatory association between miR-449a and PDGFRB, the present study performed a luciferase activity reporter assay in gastric cancer cells co-transfected with wild-type PDGFRB 3'UTR constructs and different concentrations of miR-499a mimics $(25,50$ and $100 \mathrm{nM}$ ). To verify PDGFRB as the direct target gene of miR-499a, another system transfected with mutant PDGFRB 3'UTR constructs and miR-499a mimics (100 nM) was 


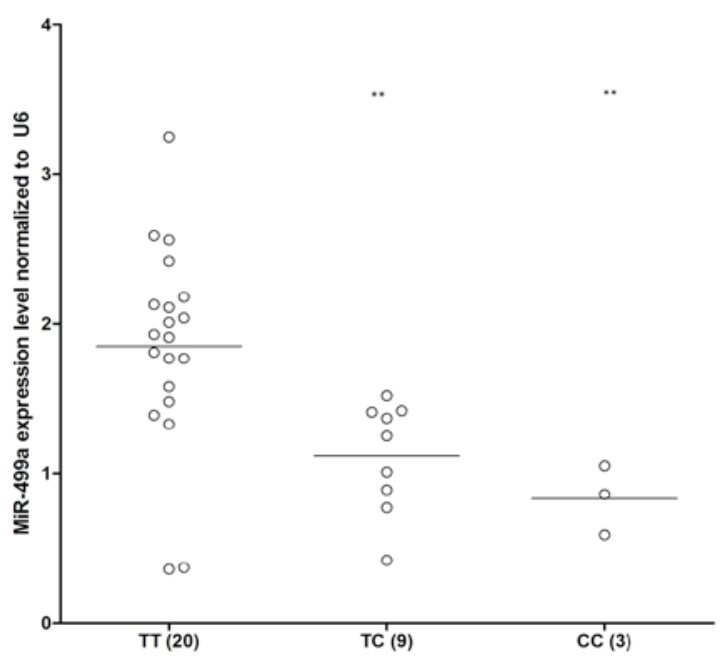

Figure 3. Expression levels of miR-499a. TT (n=20) showed higher expression levels of miR-499a, compared with TC $(n=9)$ and CC $(n=3)$, indicating that the presence of minor allele (C) in the rs3746444 polymorphism compromised the expression of miR-499a. ${ }^{* *} \mathrm{P}<0.01$ vs. control. miR, microRNA.
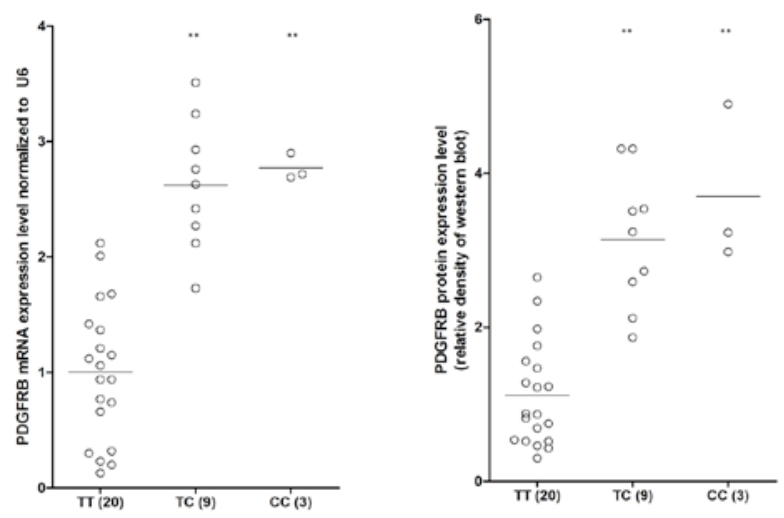

Figure 4. Levels of PDGFRB. Reverse transcription-quantitative polymerase chain reaction and western blot analyses were performed to examine the mRNA and protein expression levels of PDGFRB among different genotypes. The mRNA (left) and protein (right) expression levels of PDGFRB in the TT sample group were lower, compared with those in the minor allele-carrying groups (TC and CC). ${ }^{* *} \mathrm{P}<0.01$ vs. control. PDGFRB, platelet-derived growth factor receptor $\beta$.

examined. As shown in Fig. 2, compared with the scramble control, the relative luciferase activity of the cells transfected with the wild-type PDGFRB 3'UTR constructs decreased as the concentration of miR-499a mimics increased, exhibiting a negative regulation in a step-wise manner. By contrast, cells carrying mutant PDGFRB 3'UTR constructs exhibited a comparable relative luciferase activity index, compared with the scramble controls, indicating PDGFRB as the direct target gene of miR-499a, with the binding site located in the mutated segment.

Expression levels of miR-499a and PDGFRB vary in different genotype groups of the rs3746444 polymorphism. The rs3746444 polymorphism has previously been reported to interfere with the expression of miR-499, leading to different expression levels of miR-499a (18). Among the EGC samples collected, as shown in Fig. 3, TT $(n=20)$ showed a markedly higher expression level of miR-499a, compared with TC $(n=9)$ and $\mathrm{CC}(\mathrm{n}=3)$, indicating that the presence of minor allele (C) of the rs3746444 polymorphism compromised the expression of miR-499a.

In the present study, RT-qPCR and western blot analyses were also performed to investigate the mRNA and protein expression levels of PFGRBR among the different genotypes. As shown in Fig. 4, the mRNA and protein expression levels of PFGRBR in the TT sample group were markedly lower, compared with those in the minor allele TC and CC sample groups, indicating the negative regulatory association between miR-499a and PDGFRB.

miR-499a inhibits the expression of PDGFRB. To further confirm the hypothesis that a negative regulatory association exists between miR-499a and PDGFRB, the present study investigated the mRNA and protein expression levels of PDGFRB in EGC cells treated with $50 \mathrm{nM}$ miR-499a mimics, $100 \mathrm{nM}$ miR-499a mimics and $100 \mathrm{nM}$ miR-499a inhibitors. As shown in Fig. 5, the protein (upper panel) and mRNA (lower panel) expression levels of PDGFRB in EGC cells treated with $50 \mathrm{nM}$ miR-499a mimics appeared lower, compared with those in the scramble control, whereas those in the sample group treated with $100 \mathrm{nM}$ miR-499a mimics were lower, compared with those in the $50 \mathrm{nM}$ treatment group (Fig. 5A). This confirmed the effect of different miR-499a concentrations on the interaction between miR-499a and PDGFRB. In addition, the miR-499a inhibitor treatment group showed markedly higher protein (upper panel) and mRNA (lower panel) expression levels of PDGFRB, compared with the scramble controls and the miR-499a mimic treatment groups (Fig. 5B), confirming the negative regulatory association between miR-499a and PDGFRB.

miR-499a interferes with the viability of EGC cells. As shown in Fig. 6, the present study also investigated the relative viability of EGC cells when transfected with miR-499a mimics (50 and $100 \mathrm{nM}$ ) and miR-499a inhibitors (100 nM). The cells transfected with $100 \mathrm{nM}$ miR-499a inhibitors showed increased viability, compared with the scramble controls, whereas the cells transfected with 50/100 nM miR-499a mimics showed comparably lower viability, indicating that miR-499a negatively interfered with the viability of the EGC cells.

miR-499a rs3746444 polymorphism as a biomarker to predict recurrence following ESD in patients with EGC. To investigate the association between the rs3746444 polymorphism and the risk of recurrence in patients with EGC who have received ESD treatment, the present study enrolled 100 patients with EGC to observe the time period between ESD treatment and EGC recurrence using Cox proportional hazard model analysis. As shown in Fig. 7, when the recurrence rate was analyzed using the log rank test, the recurrence-free duration was significantly longer in the TT individuals, compared with the TC/CC individuals, indicating that the miR-499a 
A

PDGFRB

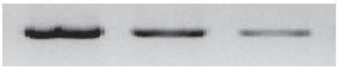

$\beta$-actin
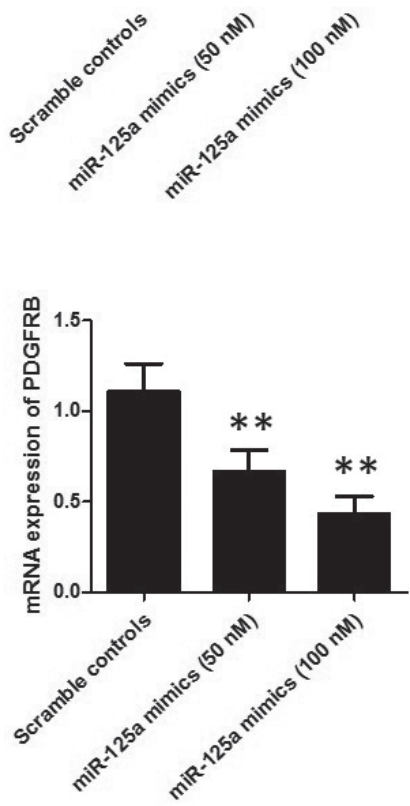

B
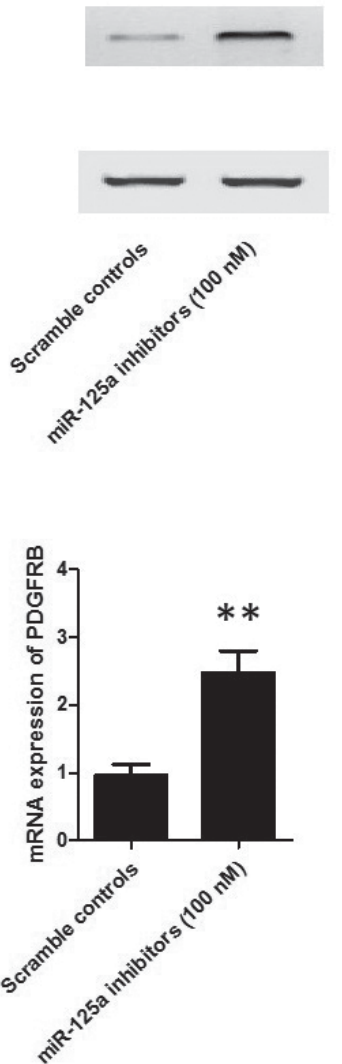

Figure 5. Confirmation of the negative regulatory association between miR-499a and PDGFRB. mRNA and protein expression levels of PDGFRB were examined in EGC cells treated with $50 \mathrm{nM}$ miR-499a mimics, $100 \mathrm{nM}$ miR-499a mimics and $100 \mathrm{nM}$ miR-499a inhibitors to confirm association between miR-499a and PDGFRB. (A) Protein (upper panel) and mRNA (lower panel) levels of PDGFRB in EGC cells treated with $50 \mathrm{nM}$ miR-499a mimics were lower, compared with those in the scramble control, whereas those in the $100 \mathrm{nM} \mathrm{miR}-499 \mathrm{a}$ mimic group were lower, compared with those in the $50 \mathrm{nM}$ treatment group. (B) Treatment with miR-499a inhibitor led to higher protein (upper panel) and mRNA (lower panel) expression levels of PDGFRB, compared with the scramble control and miR-499a mimic treatment groups. ${ }^{* *} \mathrm{P}<0.01$ vs. control. PDGFRB, platelet-derived growth factor receptor $\beta$; EGC, early gastric cancer; miR, microRNA.

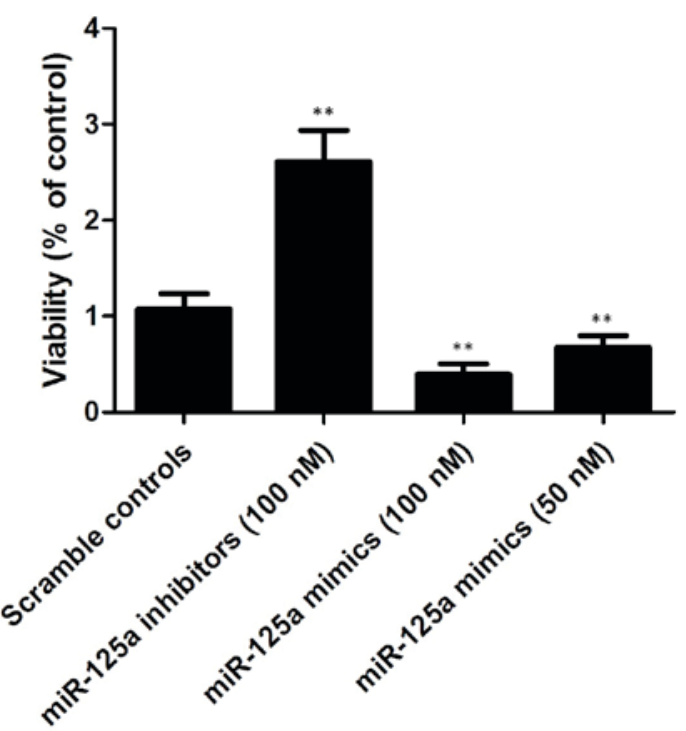

Figure 6. Viability of transfected cells. Cells transfected with $100 \mathrm{nM}$ miR-499a inhibitors showed upregulated viability, compared with scramble controls. Cells transfected with $50 \mathrm{nM} / 100 \mathrm{nM}$ miR-499a mimics showed comparably lower viability. ${ }^{* *} \mathrm{P}<0.01$ vs. control. miR, microRNA.

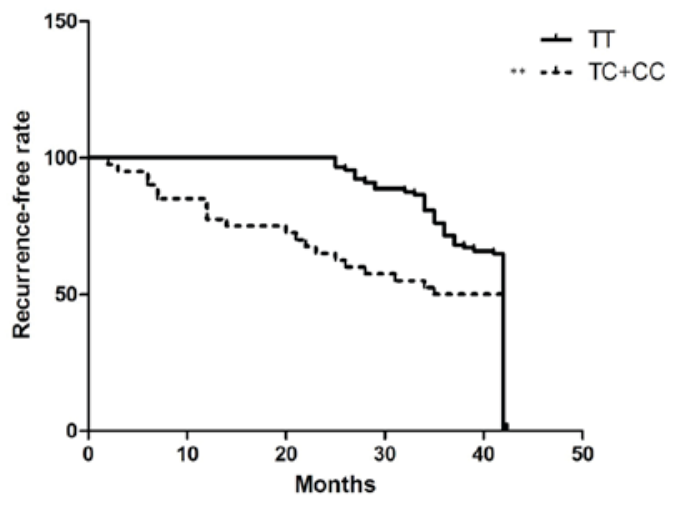

Figure 7. Recurrence-free rates. Recurrence-free rates were significantly longer in the TT genotype, compared with the TC/CC genotypes among the 100 EGC patients, indicating the miR-499a rs3746444 polymorphism may function as a biomarker to predict recurrence following endoscopic submucosal dissection in patients with EGC. ${ }^{* *} \mathrm{P}<0.01$ vs. TT group. EGC, early gastric cancer.

rs3746444 polymorphisms may function as a biomarker to predict recurrence following ESD in patients with EGC. 


\section{Discussion}

For patients with EGC, endoscopic treatment has been used as curative therapy, and the prognoses of patients with EGC has been improved by endoscopic tumor resection (19-21). Although ESD provides accurate histological diagnosis, it also enables the endoscopic curative resection of EGCs, however, it has strict criteria for small, well-differentiated adenocarcinomas with minimal submucosal invasion $(22,23)$. Only gastric cancer tissues are excised using endoscopic treatment, indicating that gastric carcinoma may recur in the patients following ESD. Therefore, a molecular diagnosis, which enables the prediction of patients at high risk for recurrence of EGC is urgently required (24). It has been demonstrated that the severity of gastric atrophy may be predictive in multiple types of gastric cancer in previous reports $(25,26)$. Although the development of EGC has previously been delineated by infection with $H$. pylori by comparing $H$. pylori-positive patients and $H$. pylori-naive-negative patients, the risk of EGC recurrence was only reduced to one-third when $H$. pylori was eradicated (27).

In the present study, it was found that the miR-499a rs3746444 polymorphism may function as a biomarker to predict recurrence following ESD in patients with EGC via analyzing the recurrence-free rate of patients with EGC with different genotypes.

Several investigations have examined the function of single nucleotide polymorphisms (SNPs) located in mature or precursor miRNAs, and how they affect progression and susceptibility in different human diseases. SNPs, which are associated with miRNA can have indirect or direct effects. SNP miRNA promoters may have indirect effects on transcription, and SNPs in mRNA may destroy or create target sites (28). SNPs may also have direct effects on mature miRNA, pre-miRNA or pri-miRNA, which may enhance or impair the function or processing of miRNA (28). It has been previous reported that the rs3746444 polymorphism, situated in the seed region of pri-miR-499a, may interfere with arm selection during the production and processing of mature miRNA, and reduce the expression of the miRNA (14). This effect of the polymorphism in its host miRNA may lead to altered risks of certain human diseases via the downstream signaling pathway or mediators. In the present study, the negative regulatory association between miR-499a and PDGFRB was established via examining the relative luciferase activity in the presence of different concentrations of miR-499a mimics. Furthermore, as the rs3746444 polymorphism has previously been reported to interfere with the expression of miR-499a, the present study investigated the expression level of different genotypes, including TT $(n=20)$, TC $(n=9)$ and $C C(n=3)$, which supported the hypothesis that the presence of minor allele $(\mathrm{C})$ of the $\mathrm{rs} 3746444$ polymorphism compromised the expression of miR-499a. The present study also performed RT-qPCR and western blot analyses to investigate the mRNA and protein expression levels of PFGRBR among different genotypes or between cells treated with different concentrations of miR-499a mimics/inhibitors. The results indicated the negative regulatory association between miR-499a and PDGFRB.

PDGFR and its ligand, PDGFm are involved in carcinogenesis and tumor development. Guo et al (15) found that the overexpression of PDGF-B and PDGFR- $\beta$ correlates with cancer progression and the lymphogenous metastasis of gastric carcinoma (15). The overexpression of PDGFR- $\alpha$ has been observed in metastatic medulloblastoma patient samples, compared with non-metastatic patient samples, in which the disruption of PDGFR- $\alpha$ function inhibited the metastatic potential of medulloblastoma cells in vitro (29). Mathey et al (30) also found that PDGFR- $\beta$ may be important as an anti-angiogenic agent and has now become a component of the standard treatment of ovarian cancer. Furthermore, the expression levels of PDGFR are associated with the angiogenesis, invasion and metastasis of colon cancer (31-33). A previous study showed significantly increased mRNA levels of PDGFR- $\beta$ in locally advanced rectal tumors, compared with the corresponding normal mucosa (34). PDGFR is considered to provide a favorable microenvironment for the growth and survival of cancer cells $(35,36)$. In a previous study, Gialeli et al (37) found that the PDGF/PDGFR axis is of paramount importance in the tumor microenvironment, and the inhibition of PDGF receptor activation represents a major target for future anticancer therapies. Therefore, the present study hypothesized that attenuating the expression of PDGFR may inhibit the growth, invasion and metastasis of tumors. The present study investigated the relative viabilities of GC cells when transfected with miR-499a mimics (50 and $100 \mathrm{nM})$ and miR-499a inhibitors (100 $\mathrm{nM})$, and found that cells transfected with $100 \mathrm{nM}$ miR-499a inhibitors showed upregulated viability, compared with the scramble control group, whereas cells transfected with 50/100 nM miR-499a mimics showed comparably lower viabilities, indicating that miR-499a negatively interfered with the viability of the EGC cells.

Taken together, the present study demonstrated that patients carrying at least one minor allele of the rs 3746444 polymorphism had a higher recurrence rate, compared with those with wild-type following ESD. The rs3746444 polymorphism, which may downregulate the expression of miR-499a and thereby upregulate the expression of PDGFRB in primary EGC, may become a useful biomarker for predicting patients at high risk of recurrence following ESD.

\section{References}

1. Correa P: International cancer epidemiology meetings. Cancer Epidemiol Biomarkers Prev 1: 245-247, 1992

2. Nagano H, Ohyama S, Fukunaga T, Seto Y, Fujisaki J, Yamaguchi T, Yamamoto N, Kato $\mathrm{Y}$ and Yamaguchi $\mathrm{A}$ : Indications for gastrectomy after incomplete EMR for early gastric cancer. Gastric Cancer 8: 149-154, 2005.

3. Ono H, Kondo H, Gotoda T, Shirao K, Yamaguchi H, Saito D, Hosokawa K, Shimoda T and Yoshida S: Endoscopic mucosal resection for treatment of early gastric cancer. Gut 48: 225-229, 2001.

4. Toyonaga T, Nishino E, Hirooka T, Ueda C and Noda $\mathrm{K}$ : Intraoperative bleeding in endoscopic submucosal dissection in the stomach and strategy for prevention and treatment. Digestive Endoscopy 18 (Suppl): S123-S127, 2006.

5. Imaeda H, Iwao Y, Ogata H, Ichikawa H, Mori M, Hosoe N, Masaoka T, Nakashita M, Suzuki H, Inoue N, et al: A new technique for endoscopic submucosal dissection for early gastric cancer using an external grasping forceps. Endoscopy 38: 1007-1010, 2006.

6. Gotoda T: Endoscopic resection of early gastric cancer. Gastric Cancer 10: 1-11, 2007.

7. Huang JQ, Sridhar S, Chen Y and Hunt RH: Meta-analysis of the relationship between Helicobacter pylori seropositivity and gastric cancer. Gastroenterology 114: 1169-1179, 1998. 
8. Parsonnet J, Friedman GD, Vandersteen DP, Chang Y, Vogelman JH, Orentreich N and Sibley RK: Helicobacter-pylori infection and the risk of gastric-carcinoma. N Engl J Med 325: 1127-1131, 1991.

9. Nomura A, Stemmermann GN, Chyou PH, Kato I, Perezperez GI and Blaser MJ: Helicobacter-pylori infection and gastric-carcinoma among Japanese-Americans in Hawaii. N Engl J Med 325: 1132-1136, 1991.

10. Ebert MS and Sharp PA: Roles for microRNAs in conferring robustness to biological processes. Cell 149: 515-524, 2012.

11. Pritchard CC, Cheng HH and Tewari M: MicroRNA profiling: Approaches and considerations. Nat Rev Genet 13: 358-369, 2012.

12. Yang JS and Lai EC: Alternative miRNA biogenesis pathways and the interpretation of core miRNA pathway mutants. Mol Cell 43: 892-903, 2011.

13. Ryan BM, Robles AI and Harris CC: Genetic variation in microRNA networks: The implications for cancer research. Nat Rev Cancer 10: 398-402, 2010.

14. Hou YY, Lee JH, Chen HC, Yang CM, Huang SJ, Liou HH, Chi CC, Tsai KW and Ger LP: The association between miR-499a polymorphism and oral squamous cell carcinoma progression. Oral Dis 21: 195-206, 2015.

15. Guo Y, Yin J, Zha L and Wang Z: Clinicopathological significance of platelet-derived growth factor B, platelet-derived growth factor receptor- $\beta$ and $\mathrm{E}$-cadherin expression in gastric carcinoma. Contemp Oncol (Pozn) 17: 150-155, 2013.

16. Livak KJ and Schmittgen TD: Analysis of relative gene expression data using real-time quantitative PCR and the 2(-Delta Delta C(T)) Method. Methods 25: 402-408, 2001.

17. Sachinidis A, Skach RA, Seul C, Ko Y, Hescheler J, Ahn HY and Fingerle J: Inhibition of the PDGF beta-receptor tyrosine phosphorylation and its downstream intracellular signal transduction pathway in rat and human vascular smooth muscle cells by different catechins. FASEB J 16: 893-895, 2002.

18. Liu J, Xie B, Chen S, Jiang F and Meng W: Association study of two inflammation-related polymorphisms with susceptibility to hepatocellular carcinoma: A meta-analysis. BMC Med Genet 15: 92, 2014

19. Amano Y, Ishihara S, Amano K, Hirakawa K, Adachi K, Fukuda R, Watanabe M, Fukumoto S, Fujishiro H and Imaoka T: An assessment of local curability of endoscopic surgery in early gastric cancer without satisfaction of current therapeutic indications. Endoscopy 30: 548-552, 1998.

20. Sano T, Kobori O and Muto T: Lymph node metastasis from early gastric cancer: Endoscopic resection of tumour. Br J Surg 79: 241-244, 1992

21. Haruma K, Sumii K, Inoue K, Teshima H and Kajiyama G: Endoscopic therapy in patients with inoperable early gastric cancer. Am J Gastroenterol 85: 522-526, 1990

22. Kato M: Endoscopic submucosal dissection (ESD is being accepted as a new procedure of endoscopic treatment of early gastric cancer. Intern Med 44: 85-86, 2005.

23. Hirasaki S, Tanimizu M, Nasu J, Shinji T and Koide N: Treatment of elderly patients with early gastric cancer by endoscopic submucosal dissection using an insulated-tip diathermic knife. Intern Med 44: 1033-1038, 2005.

24. Miyoshi E, Haruma K, Hiyama T, Tanaka S, Yoshihara M, Shimamoto $\mathrm{F}$ and Chayama K: Microsatellite instability is a genetic marker for the development of multiple gastric cancers. Int J Cancer 95: 350-353, 2001
25. Hanaoka N, Uedo N, Shiotani A, Inoue T, Takeuchi Y, Higashino $\mathrm{K}$, Ishihara $\mathrm{R}$, Iishi $\mathrm{H}$, Haruma $\mathrm{K}$ and Tatsuta $\mathrm{M}$ : Autofluorescence imaging for predicting development of metachronous gastric cancer after Helicobacter pylori eradication. J Gastroenterol Hepatol 25: 1844-1849, 2010.

26. Shiotani A, Uedo N, Iishi H, Yoshiyuki Y, Ishii M, Manabe N, Kamada T, Kusunoki H, Hata J and Haruma K: Predictive factors for metachronous gastric cancer in high-risk patients after successful Helicobacter pylori eradication. Digestion 78 : 113-119, 2008.

27. Fukase K, Kato M, Kikuchi S, Inoue K, Uemura N, Okamoto S, Terao S, Amagai K, Hayashi S and Asaka M; Japan Gast Study Group: Effect of eradication of Helicobacter pylori on incidence of metachronous gastric carcinoma after endoscopic resection of early gastric cancer: An open-label, randomised controlled trial. Lancet 372: 392-397, 2008.

28. Salzman DW and Weidhaas JB: SNPing cancer in the bud: microRNA and microRNA-target site polymorphisms as diagnostic and prognostic biomarkers in cancer. Pharmacol Ther 137: $55-63,2013$.

29. MacDonald TJ, Brown KM, LaFleur B, Peterson K, Lawlor C, Chen Y, Packer RJ, Cogen P and Stephan DA: Expression profiling of medulloblastoma: PDGFRA and the RAS/MAPK pathway as therapeutic targets for metastatic disease. Nat Genet 29: 143-152, 2001.

30. Mathey S, Graeser MK, Zu Eulenburg C, Woelber L, Trillsch F, Jaenicke F, Müller V, Milde-Langosch K and Mahner S: Platelet-derived growth factor receptor beta serum concentrations during first-line therapy in ovarian cancer. Oncology 85: 69-77, 2013.

31. Kitadai Y, Sasaki T, Kuwai T, Nakamura T, Bucana CD, Hamilton SR and Fidler IJ: Expression of activated platelet-derived growth factor receptor in stromal cells of human colon carcinomas is associated with metastatic potential. Int J Cancer 119: 2567-2574, 2006.

32. Mancuso MR, Davis R, Norberg SM, O'Brien S, Sennino B, Nakahara T, Yao VJ, Inai T, Brooks P, Freimark B, et al: Rapid vascular regrowth in tumors after reversal of VEGF inhibition. J Clin Invest 116: 2610-2621, 2006

33. Song S, Ewald AJ, Stallcup W, Werb Z and Bergers G PDGFRbeta+ perivascular progenitor cells in tumours regulate pericyte differentiation and vascular survival. Nat Cell Biol 7: 870-879, 2005

34. Erben P, Horisberger K, Muessle B, Müller MC, Treschl A, Ernst T, Kähler G, Ströbel P, Wenz F, Kienle P, et al: mRNA expression of platelet-derived growth factor receptor-beta and C-KIT: Correlation with pathologic response to cetuximab-based chemoradiotherapy in patients with rectal cancer. Int J Radiat Oncol Biol Phys 72: 1544-1550, 2008.

35. Liotta LA and Kohn EC: The microenvironment of the tumour-host interface. Nature 411: 375-379, 2001.

36. Radinsky R and Fidler IJ: Regulation of tumor cell growth at organ-specific metastases. In Vivo 6: 325-331, 1992.

37. Gialeli C, Nikitovic D, Kletsas D, Theocharis AD, Tzanakakis GN and Karamanos NK: PDGF/PDGFR signaling and targeting in cancer growth and progression: Focus on tumor microenvironment and cancer-associated fibroblasts. Curr Pharm Des 20: 2843-2848, 2014 